\title{
Public Service Obligations for Air Transport in the United States and Europe: \\ Connectivity Effects and Value for Money
}

\section{Michael D. Wittman ${ }^{A} /$ Florian Allroggen ${ }^{B} /$ Robert Malina $^{B, C}$}

${ }^{A}$ MIT International Center for Air Transportation, Cambridge, MA

${ }^{B}$ MIT Laboratory for Aviation and the Environment, Cambridge, MA

${ }^{C}$ Center for Environmental Sciences, Hasselt University, Belgium

Delft, September 162016 
Outline

\section{What are Public Service Obligations (PSOs)?}

To what extent do specific PSO's contribute to connectivity?

How much public money is being spent on PSO's and how does this compare to the connectivity impacts of those routes? 


\section{Motivation: PSO}

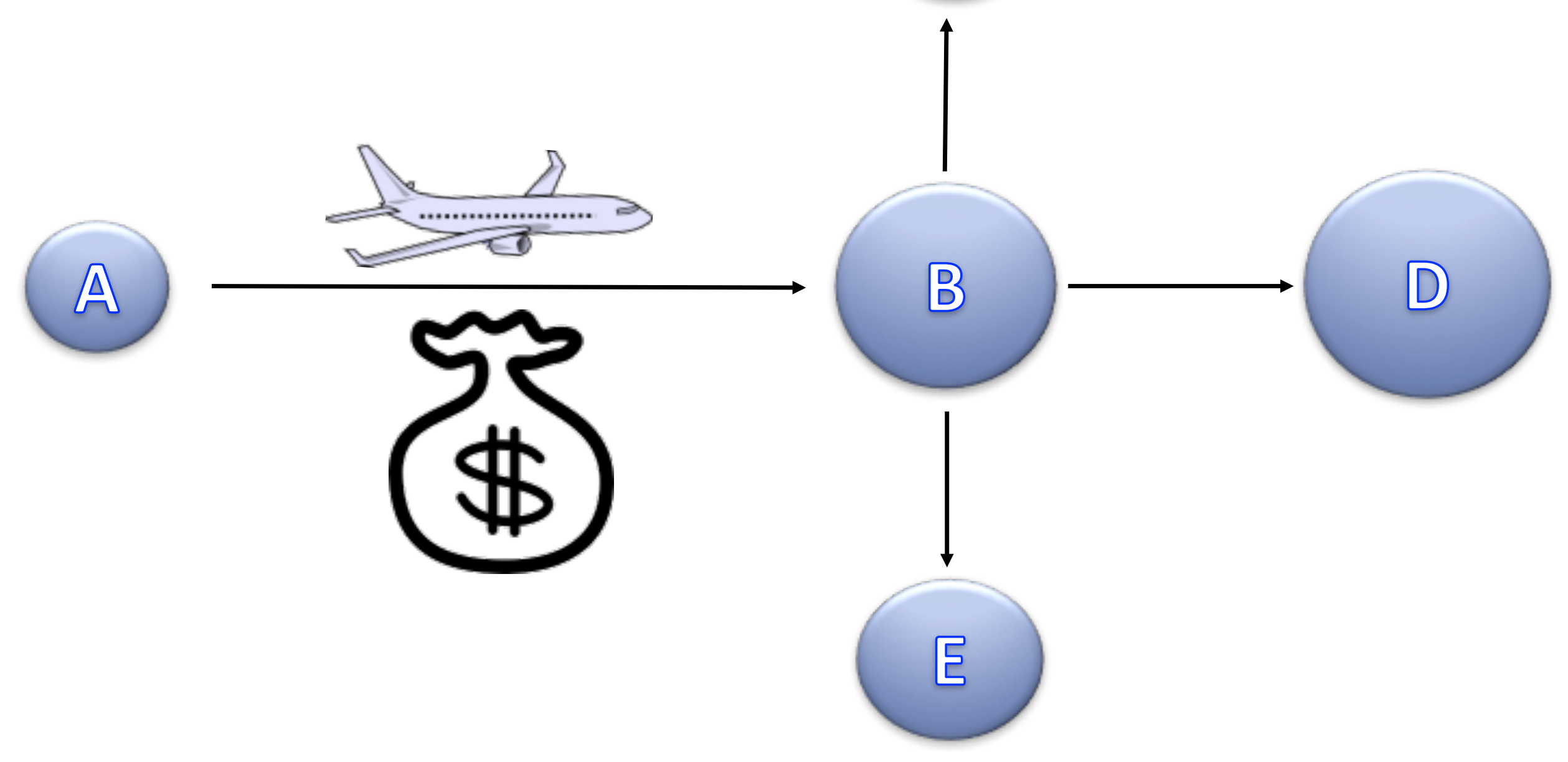

\section{C}




\section{Geographical scope}

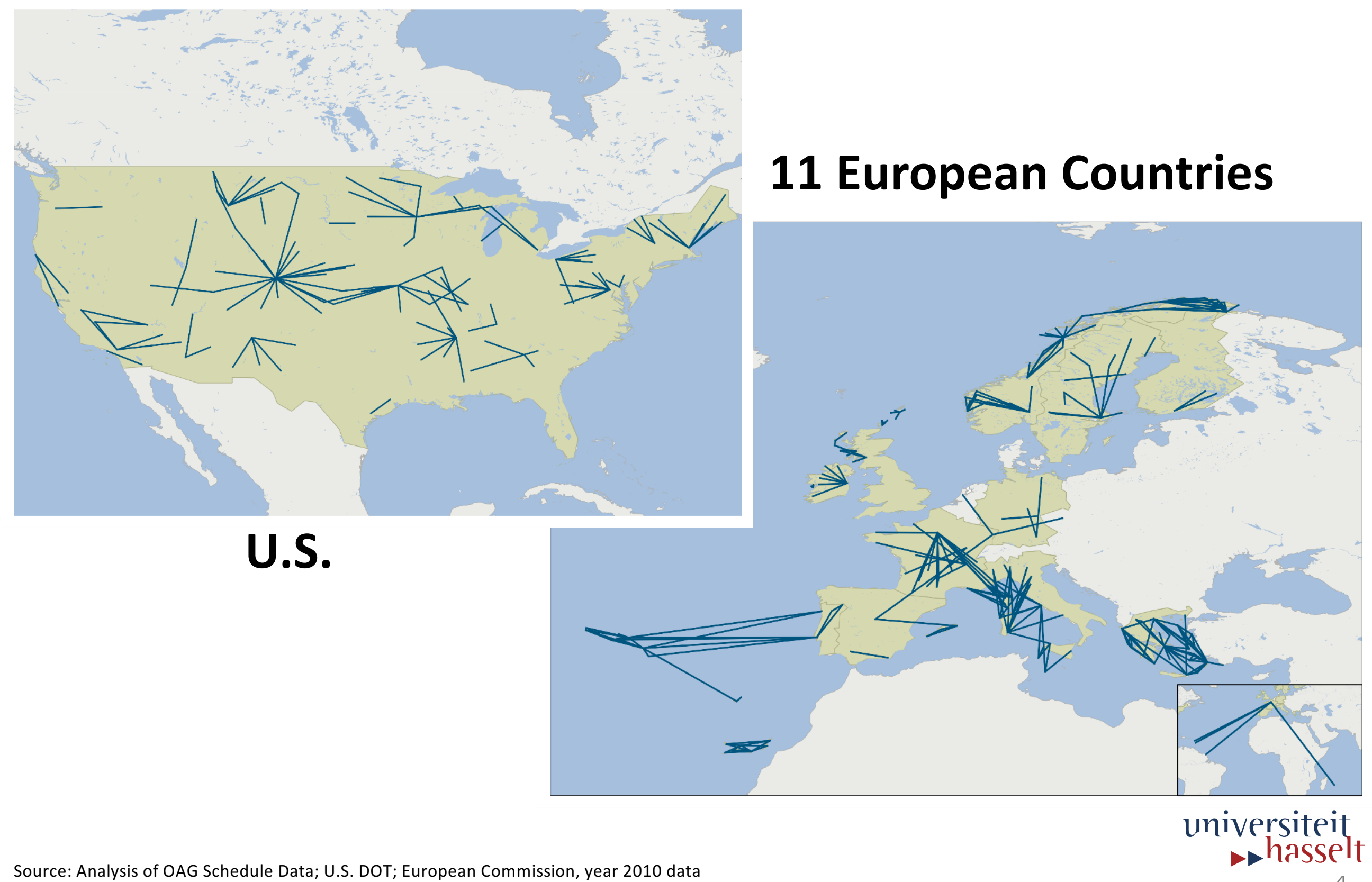


PSOs Represent about 2.5\% of Scheduled Movements in the 12 Countries Analyzed

Summary Statistics for PSO Movements in the U.S. and Europe, 2010

\begin{tabular}{|c|c|c|c|c|c|c|c|}
\hline \multirow{2}{*}{ Country } & \multirow{2}{*}{$\begin{array}{c}\text { Airports } \\
\text { with PSO } \\
\text { Flights }\end{array}$} & \multicolumn{3}{|c|}{$\begin{array}{l}\text { Thousands of Scheduled } \\
\text { Movements (2010) }\end{array}$} & \multicolumn{3}{|c|}{$\begin{array}{l}\text { Millions of Scheduled Seats } \\
\qquad(2010)\end{array}$} \\
\hline & & PSO & Total & $\begin{array}{c}\text { PSO } \\
\% \text { of Total }\end{array}$ & PSO & Total & $\begin{array}{c}\text { PSO } \\
\% \text { of Total }\end{array}$ \\
\hline Finland & 4 & 2 & 181 & $0.9 \%$ & 0.1 & 20 & $0.4 \%$ \\
\hline France & 38 & 43 & 1,074 & $4.0 \%$ & 6.7 & 155 & $4.3 \%$ \\
\hline Germany & 5 & 3 & 1,583 & $0.2 \%$ & 0.1 & 226 & $<0.1 \%$ \\
\hline Greece & 31 & 15 & 256 & $5.9 \%$ & 0.8 & 34 & $2.5 \%$ \\
\hline Ireland & 11 & 10 & 194 & $5.1 \%$ & 0.8 & 31 & $2.6 \%$ \\
\hline Italy & 13 & 32 & 1,018 & $3.1 \%$ & 5.6 & 153 & $3.7 \%$ \\
\hline Norway & 37 & 58 & 392 & $14.8 \%$ & 2.2 & 44 & $5.0 \%$ \\
\hline Portugal & 15 & 21 & 234 & $8.8 \%$ & 1.9 & 33 & $5.8 \%$ \\
\hline Spain & 14 & 90 & 1,231 & $7.3 \%$ & 6.4 & 191 & $3.3 \%$ \\
\hline Sweden & 13 & 7 & 304 & $2.3 \%$ & 0.3 & 34 & $1.0 \%$ \\
\hline U.K. & 27 & 9 & 1,662 & $0.5 \%$ & 0.1 & 252 & $0.1 \%$ \\
\hline U.S. (mainland) & 135 & 164 & 10,073 & $1.6 \%$ & 3.1 & 1,041 & $0.3 \%$ \\
\hline Total & 343 & 453 & 18,202 & $2.5 \%$ & 28.2 & 2,214 & $1.3 \%$ \\
\hline
\end{tabular}




\section{The Global Connectivity Index (Allroggen, Wittman, Malina 2015)}

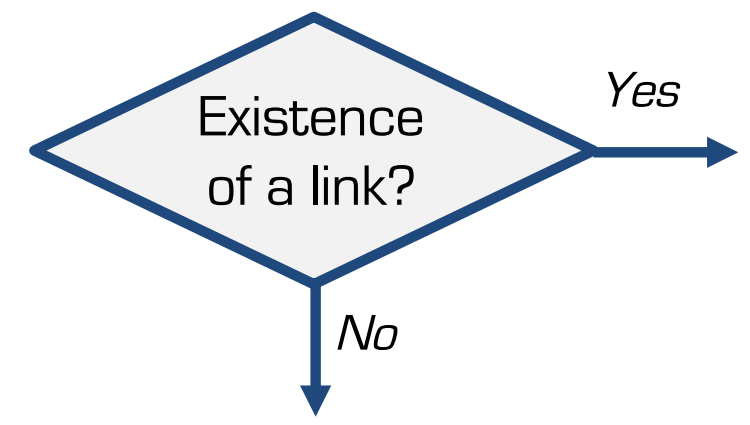

No connectivity

\section{Link-Identification-Level}

- Data source: OAG schedules 1990-2012

- Identify nonstop flights

- Onestop itinerary generator:

- Minimum connecting time

- Feasible airline combinations 


\section{The Global Connectivity Index}

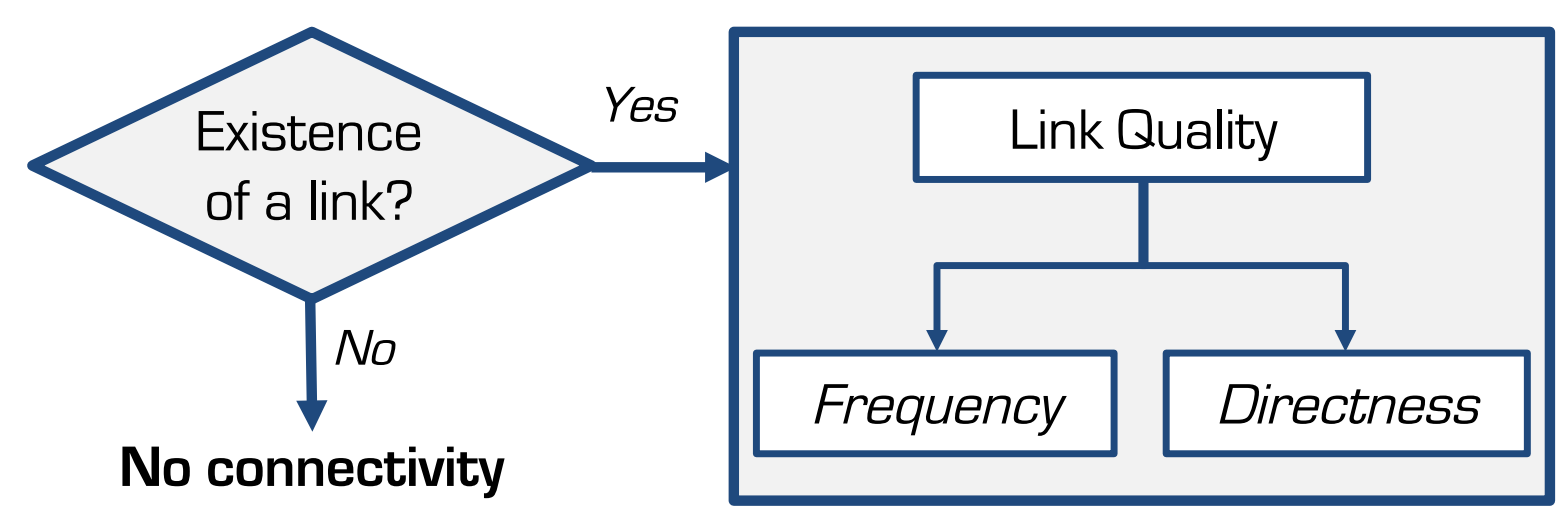

Link-Identification-Level

Link-Quality-Level

- Frequency: Counter of ops.

- Directness: Valuation of detours: The higher the relative mark-up of travel time compared to a [hypothetical] non-stop routing, the lower the link quality 


\section{The Global Connectivity Index}

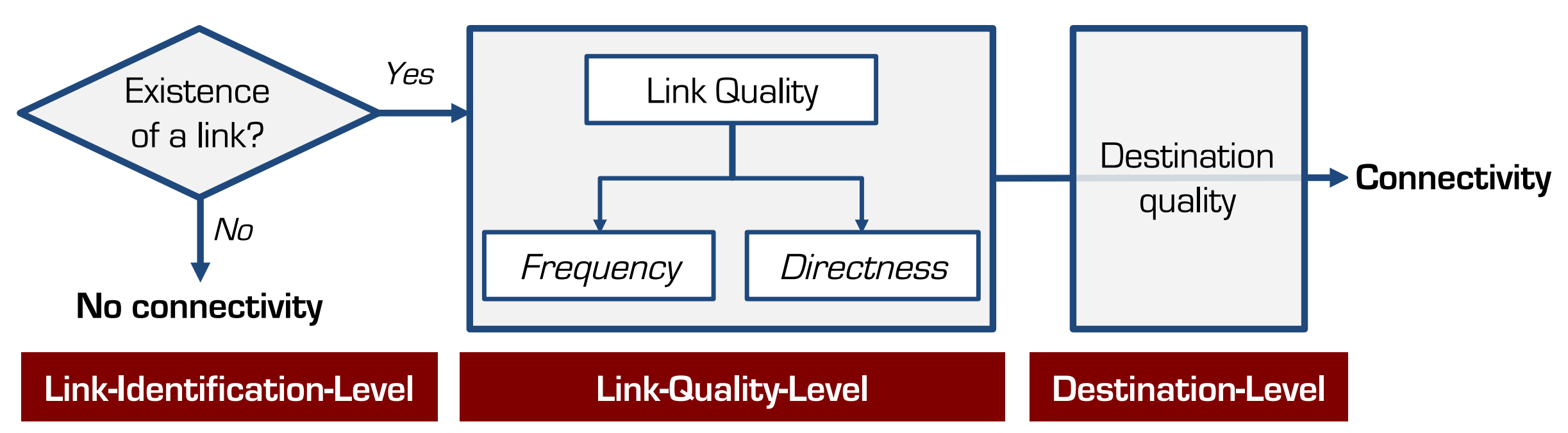

- Define and calculate metric for interaction potential: GDP

- Define geographical market of airport: distance decay modeling 
"I I InI I"
The Global Connectivity Index

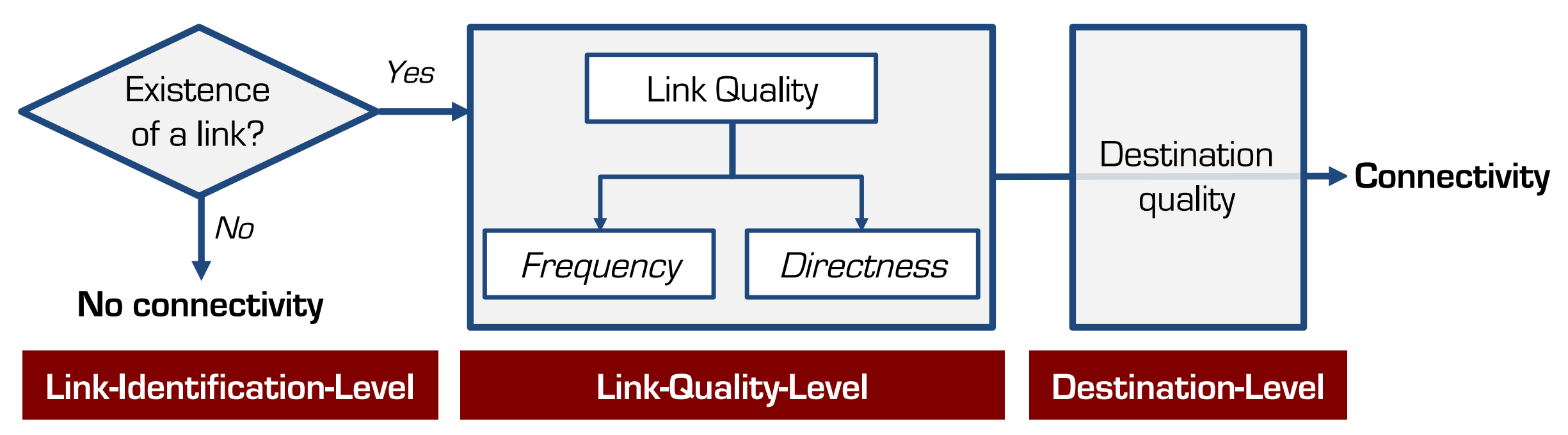




\section{Connectivity metric: Sample visualizations}

Total connectivity and share of onestop connectivity at European airports:

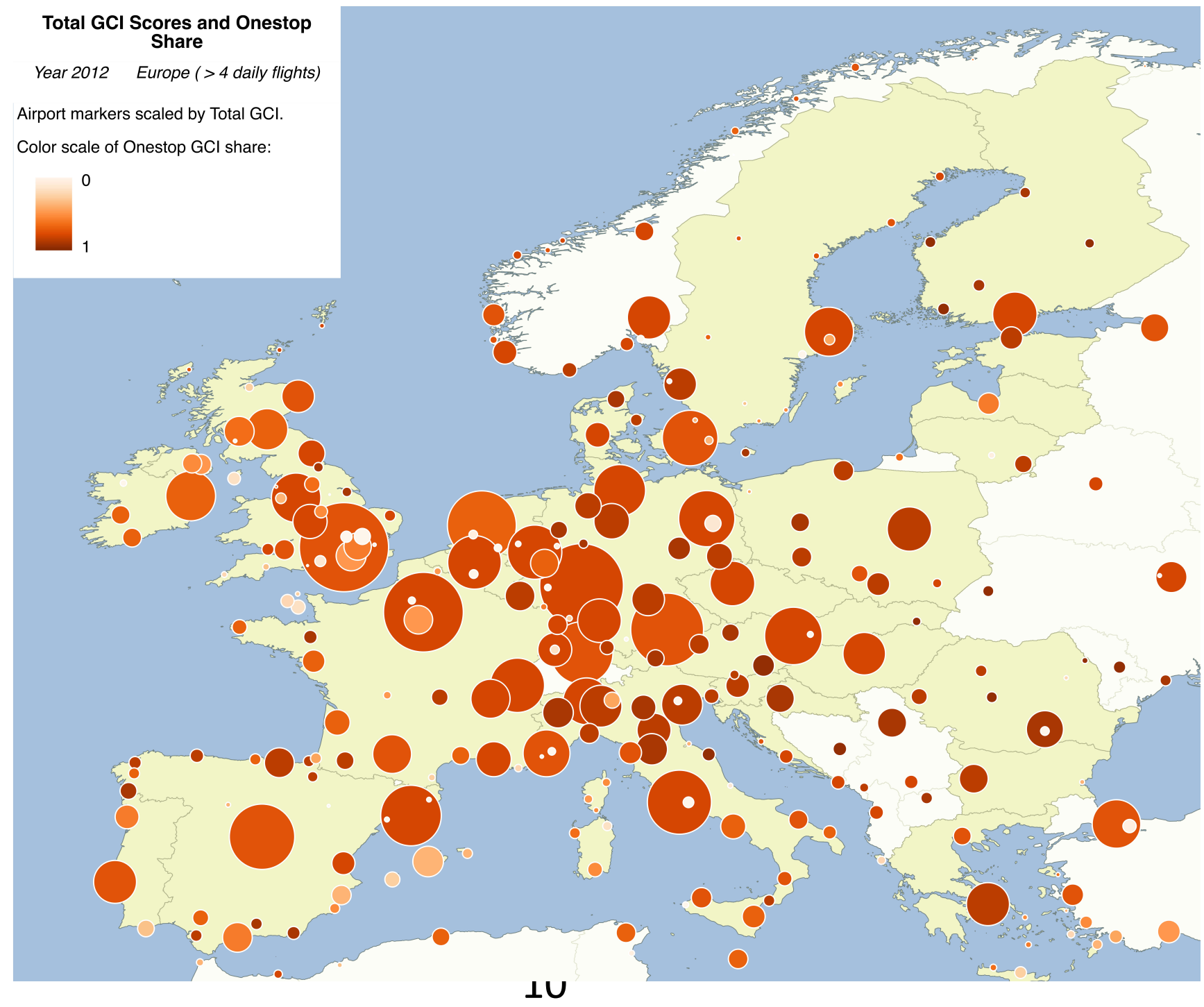




\section{Connectivity metric: Sample visualizations}

The global onestop connectivity landscape

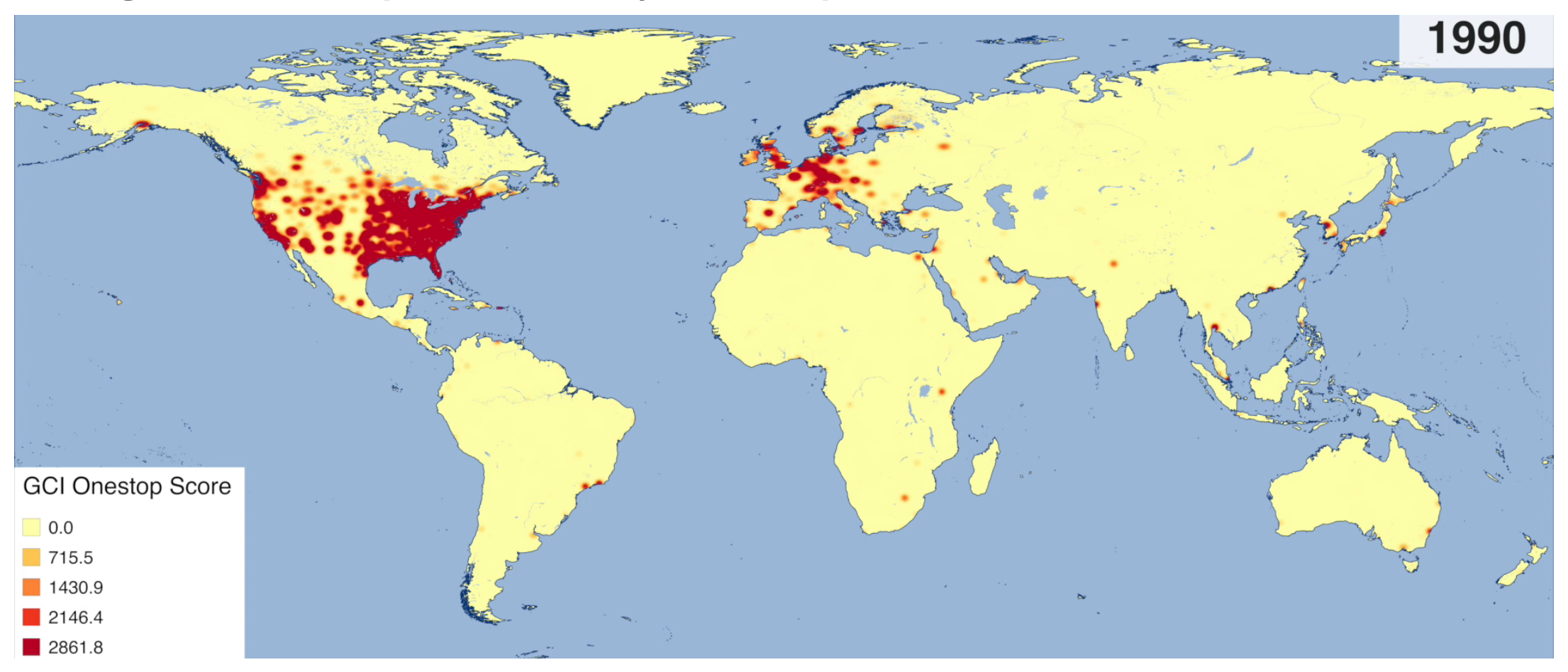




\section{Connectivity metric: Sample visualizations}

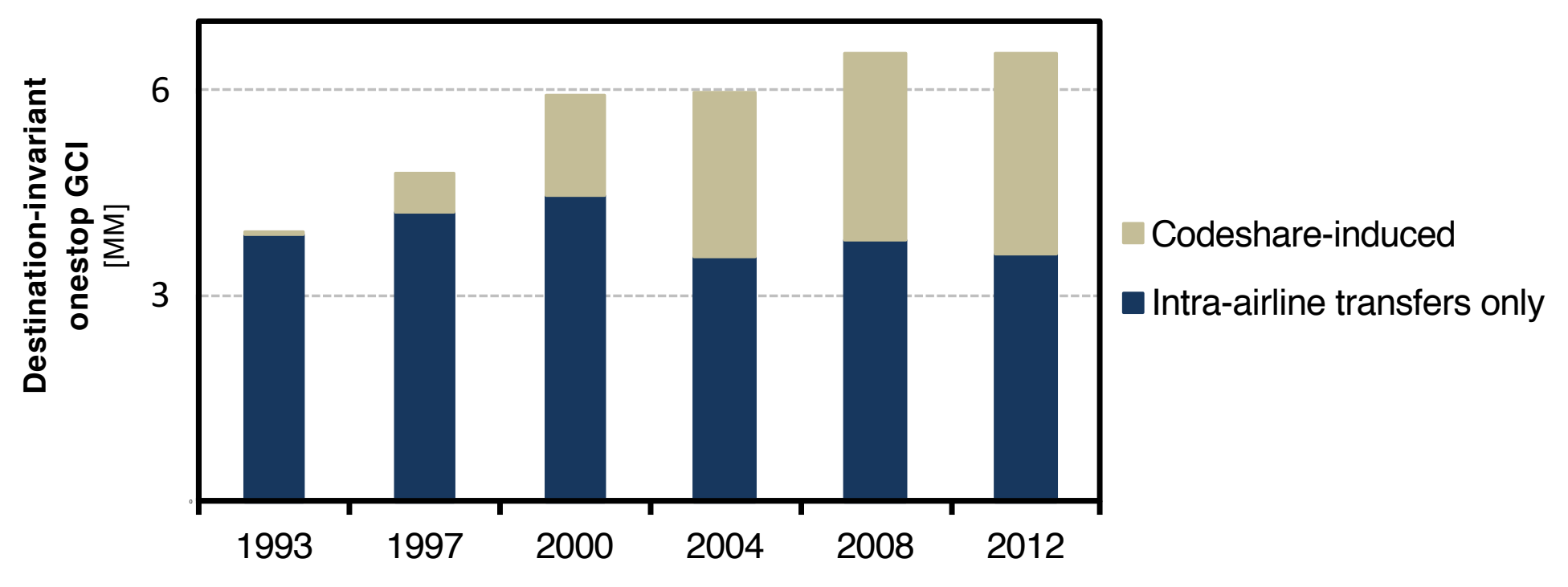


Calculation of the connectivity impacts of PSOs

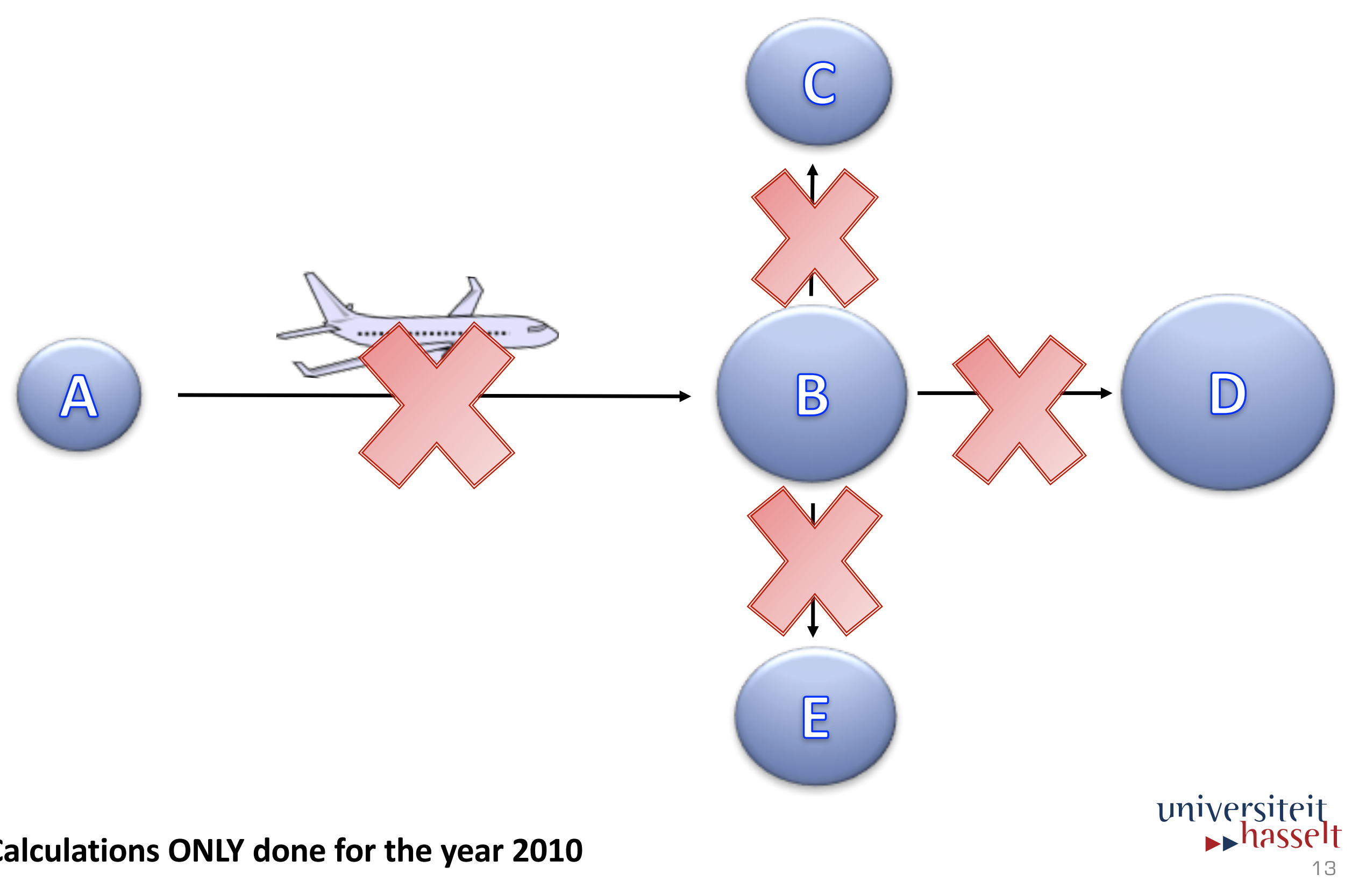


" ' In'I" the U.S.', PSO Routes Provide Limited Amounts of Direct Connectivity

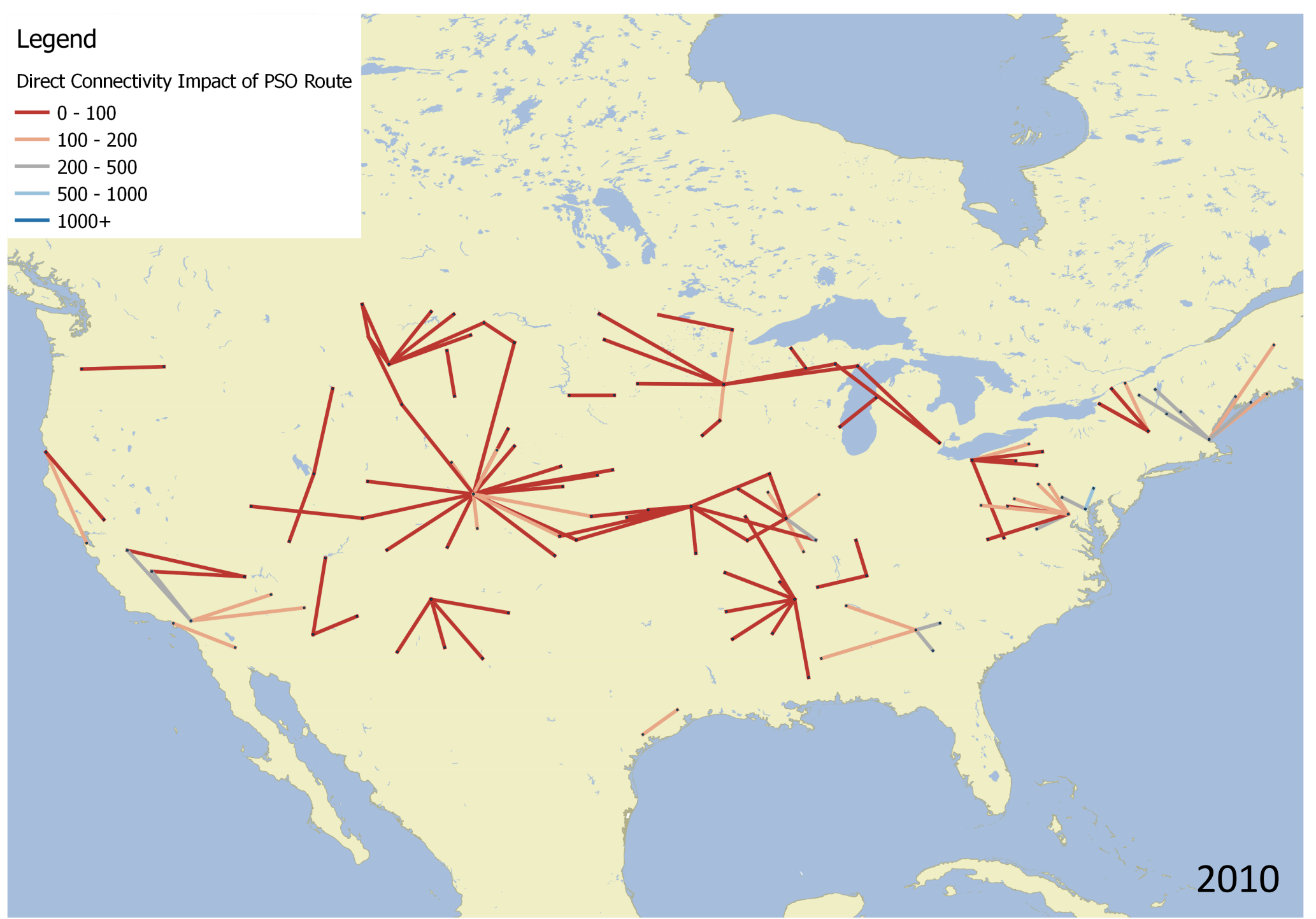


But PSOs Serving Network Carrier Hubs Can Generate Significant Indirect Connectivity

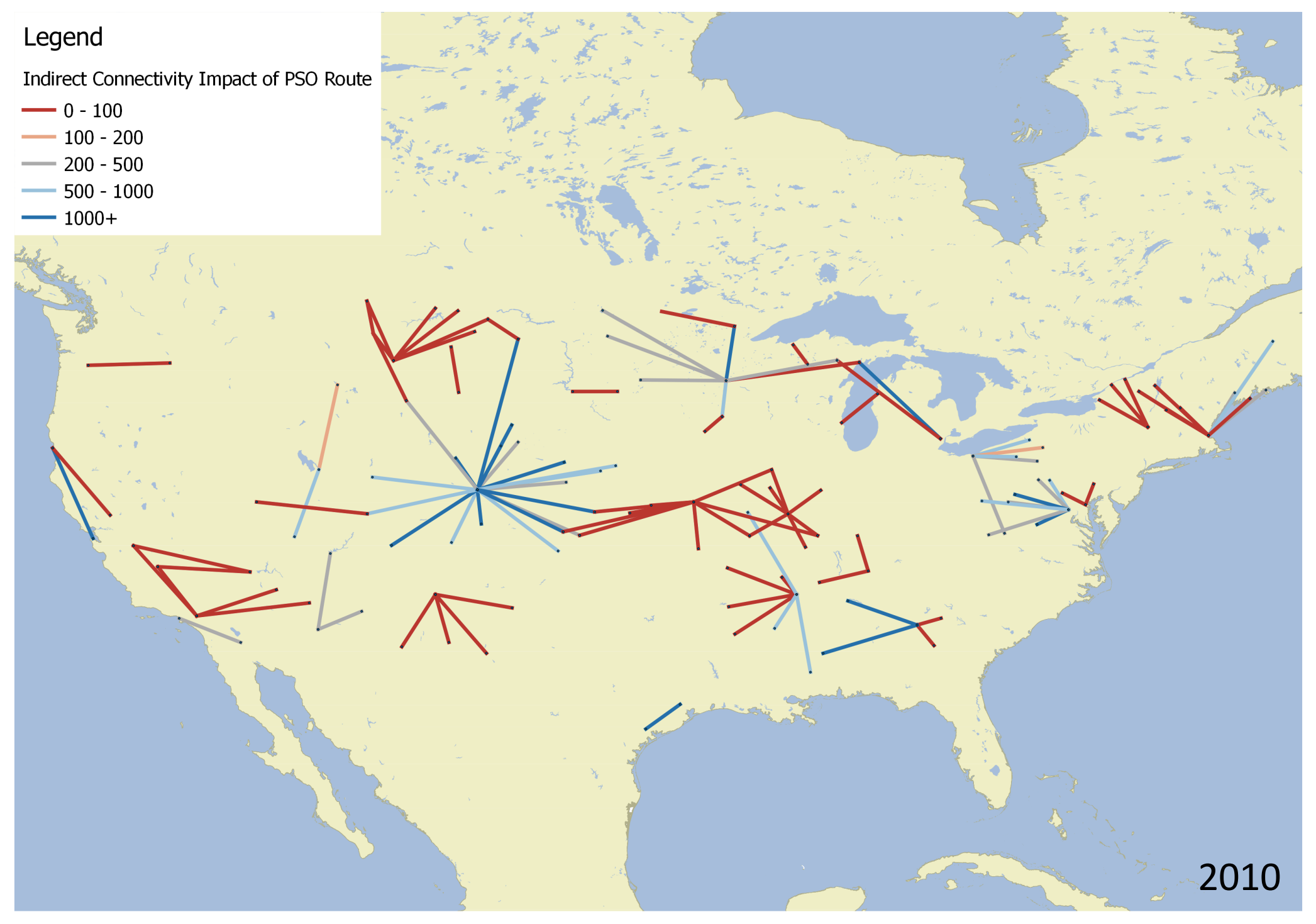




\section{Most European PSOs Provide Limited Direct Connectivity}

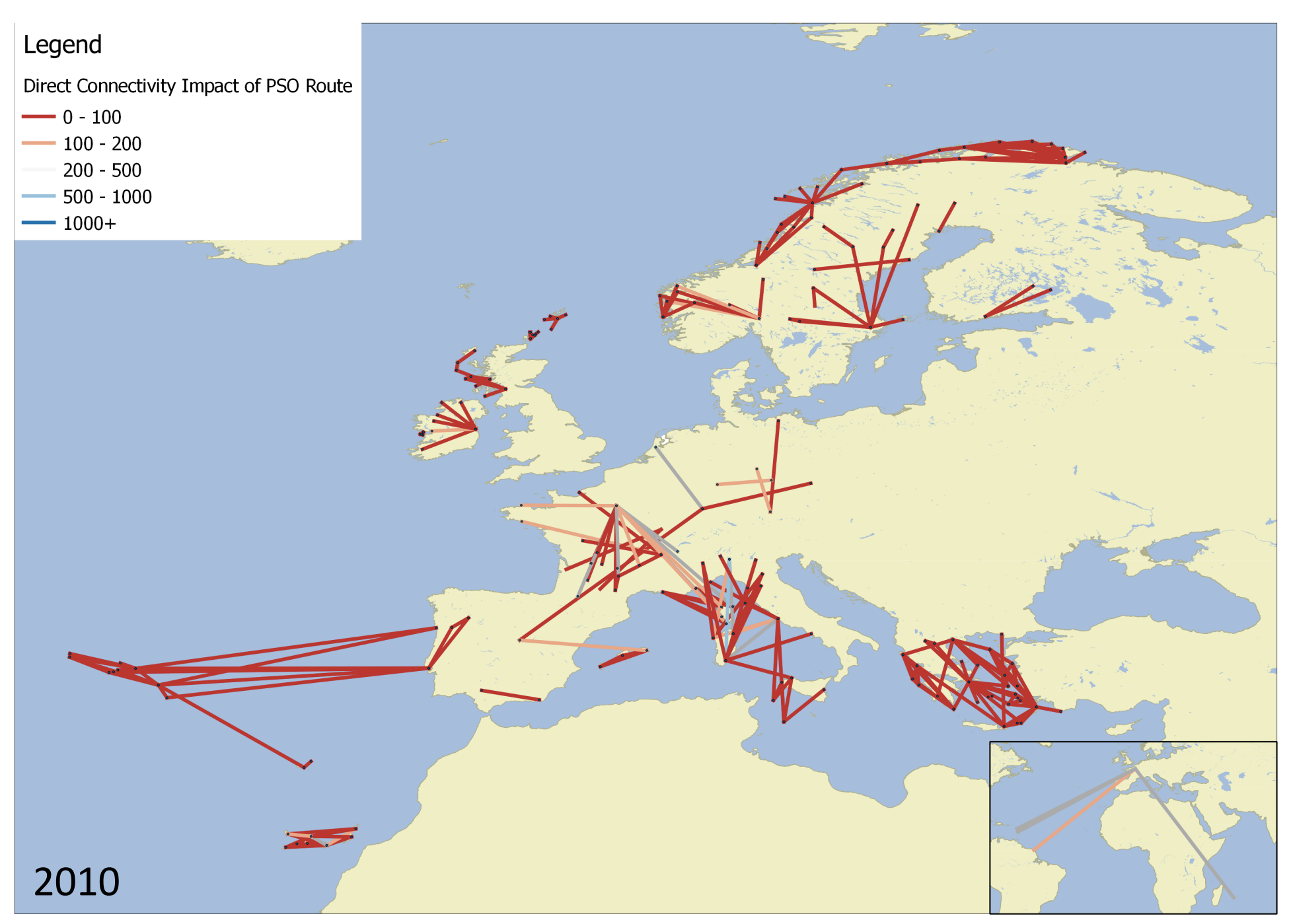




\section{Indirect Connectivity Limited for Many "Lifeline" PSO Routes, Even Those Serving Large Airports}

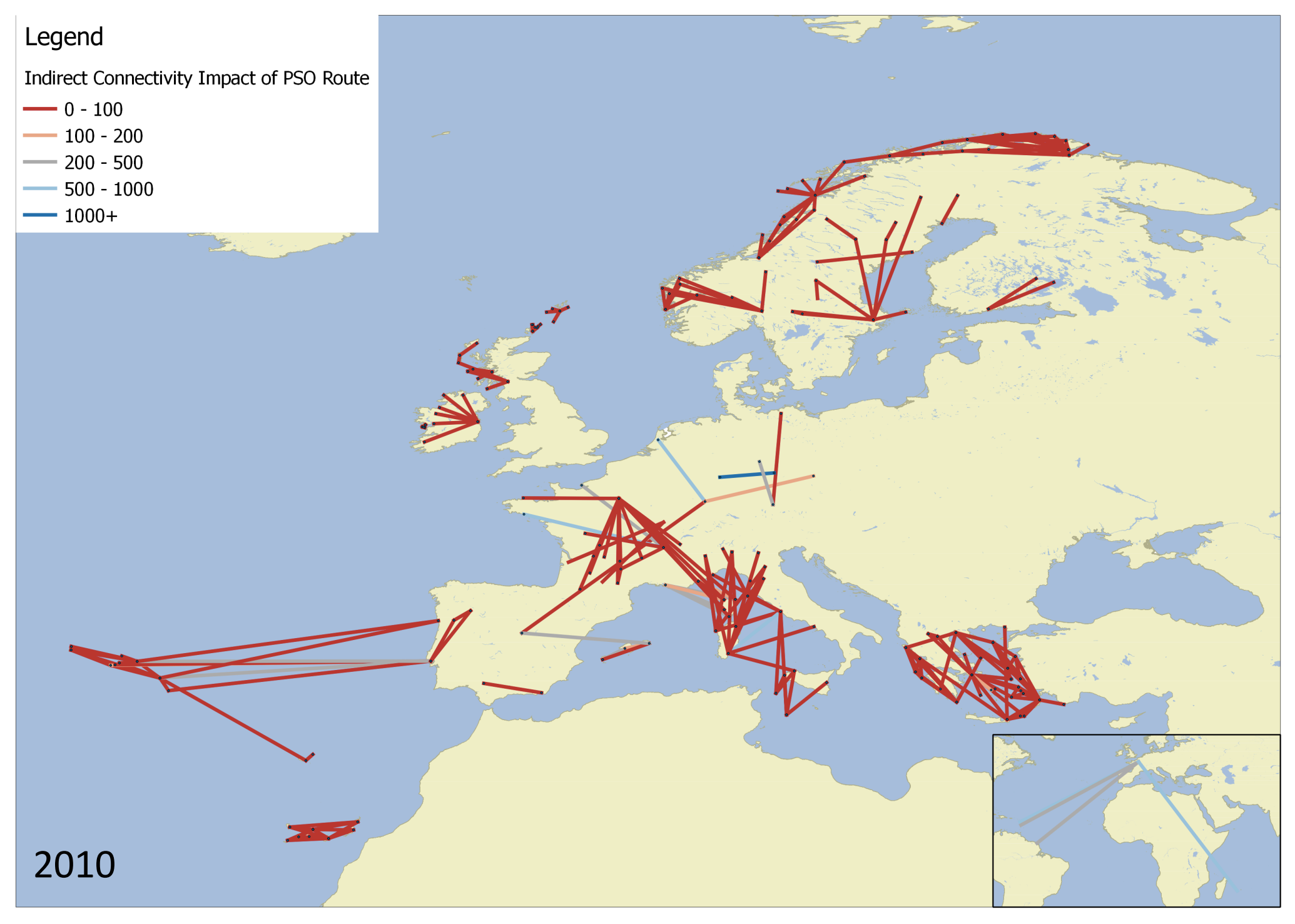


Yet PSOs are Still Critically Important; PSOs Provided All Connectivity at 142 Airports

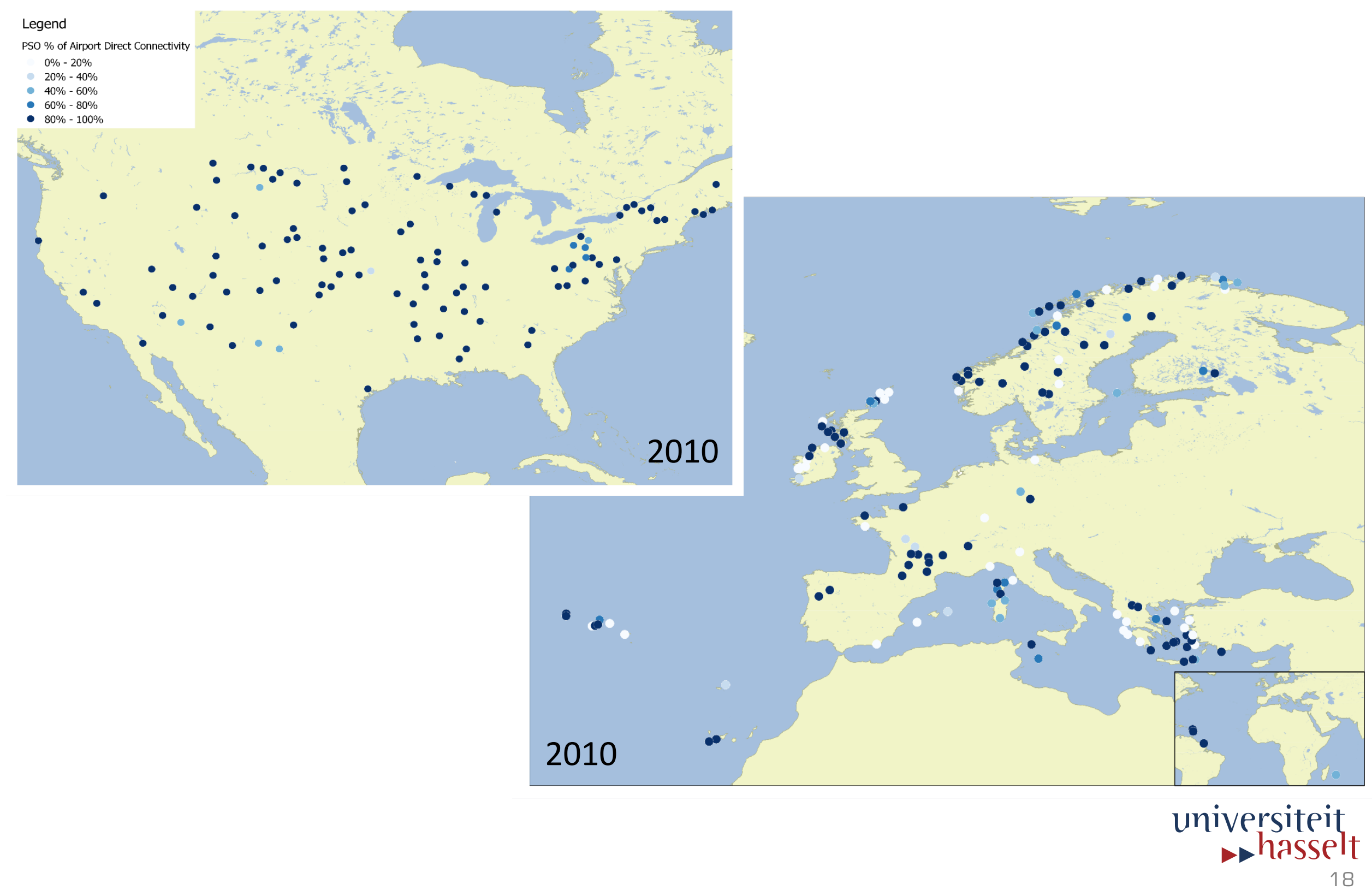




\section{A New Subsidy Dataset Provides Additional Insight into PSO Performance}

- Our PSO subsidy data set, covering $90 \%$ of all PSO movements in 2010, was collected from different sources:

\section{Academic articles}

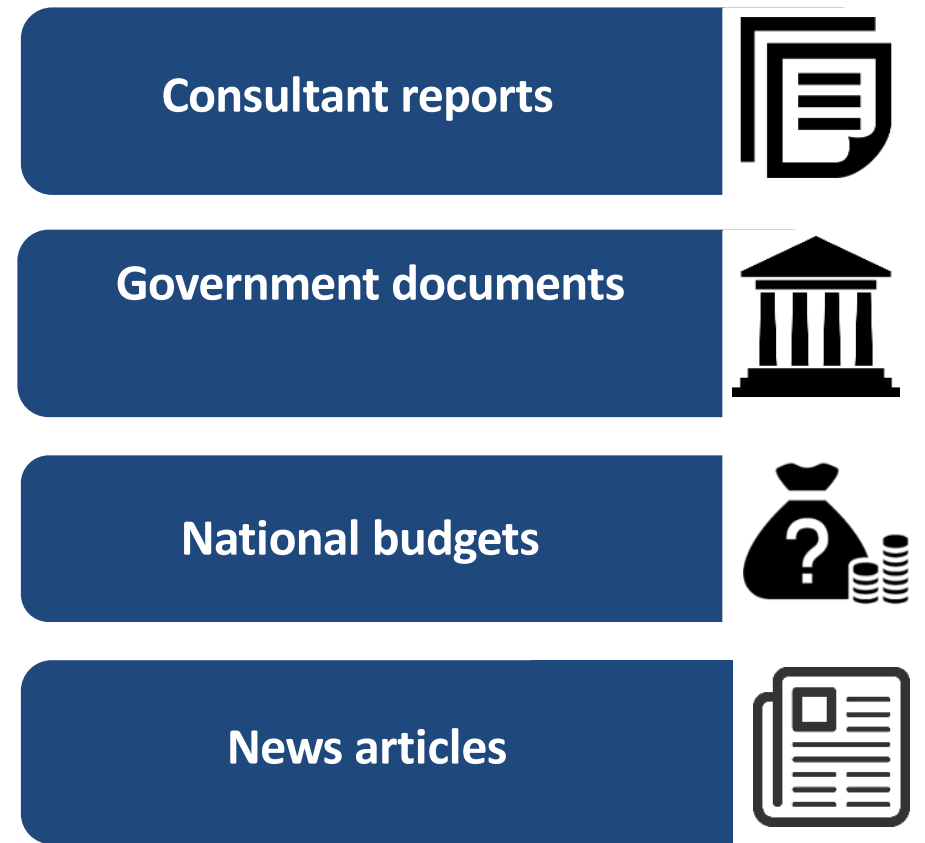




\section{Governments Earmarked Over $\$ 900$ Million for Subsidies in 2010}

\begin{tabular}{|c|c|c|c|c|}
\hline \multirow{2}{*}{ Country } & \multirow{2}{*}{$\begin{array}{l}\text { Total Annual } \\
\text { Subsidy } \\
\text { (Millions of USD, } \\
2010 \text { PPP) }\end{array}$} & \multicolumn{2}{|c|}{$\begin{array}{l}\text { Thousands of PSO } \\
\text { Movements (2010) }\end{array}$} & \multirow{2}{*}{$\begin{array}{c}\text { \% of PSO Movements } \\
\text { Covered by Subsidy } \\
\text { Data }\end{array}$} \\
\hline & & $\begin{array}{c}\text { Subsidy data } \\
\text { available }\end{array}$ & $\begin{array}{c}\text { All PSO } \\
\text { movements }\end{array}$ & \\
\hline Finland & $\$ 2.3$ & 2 & 2 & $100 \%$ \\
\hline France* & $\$ 19.6$ & 22 & 43 & $51 \%$ \\
\hline Germany & $\$ 8.3$ & 3 & 3 & $100 \%$ \\
\hline Greece* & $\$ 49.8$ & 14 & 15 & $92 \%$ \\
\hline Ireland & $\$ 19.1$ & 10 & 10 & $100 \%$ \\
\hline Italy & Not Available & 0 & 32 & $0 \%$ \\
\hline Norway & $\$ 73.3$ & 58 & 58 & $100 \%$ \\
\hline Portugal & $\$ 64.7$ & 21 & 21 & $100 \%$ \\
\hline Spain** & $\$ 495.2$ & 206 & 206 & $100 \%$ \\
\hline Sweden & $\$ 9.1$ & 7 & 7 & $100 \%$ \\
\hline U.K. & $\$ 6.2$ & 9 & 9 & $100 \%$ \\
\hline United States & $\$ 155.6$ & 164 & 164 & $100 \%$ \\
\hline Total & $\$ 903.2$ & 515 & 569 & $90 \%$ \\
\hline
\end{tabular}




\section{Average Subsidies per Movement Were of the Same Order of Magnitude Across Countries}

PSO Subsidy Amount per PSO Movement, 2010

$\$ 8,000$

$\$ 7,000$

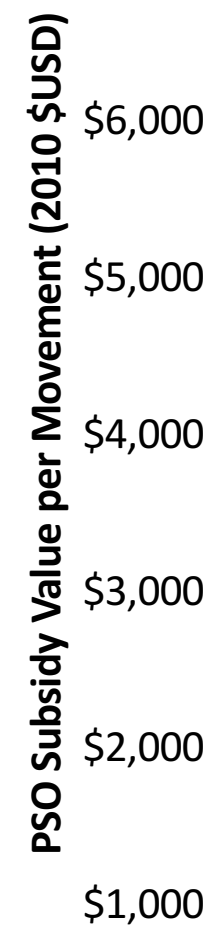

$\$ 0$

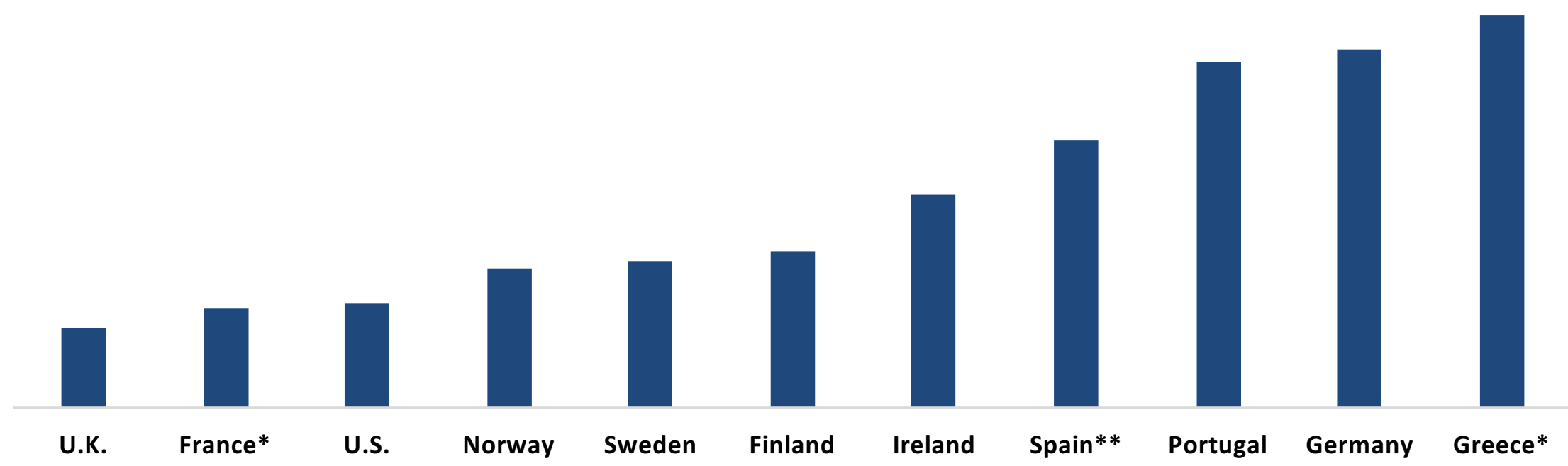

* Only routes with subsidy data available are included ( $92 \%$ of Greek movements and $51 \%$ of French movements)

** Spain data includes air discount scheme routes between the mainland and the Canary and Balearic Islands Note: Local currencies converted to USD PPP via World Bank data universiteit $\leadsto$ hasselt 


\section{Yet Programs that Focused on Indirect Connectivity Provided the Best "Bang-for-Buck"}

\section{PSO Subsidy Cost per Movement and Connectivity Point, 2010}

- Per Movement $\quad$ Per Total GCl Point

\section{$\$ 90,000$}

$\$ 80,000$

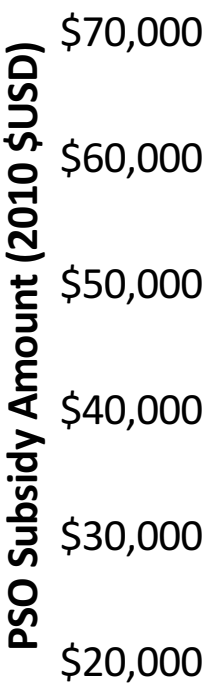

$\$ 10,000$

\section{U.S. France* Germany Spain** U.K.}

Ireland

Finland

Portugal

* Only routes with subsidy data available are included ( $92 \%$ of Greek movements and $51 \%$ of French movements)

** Spain data includes air discount scheme routes between the mainland and the Canary and Balearic Islands Note: Local currencies converted to USD PPP via World Bank data 


\section{Conclusions}

- Our analysis of PSO connectivity and subsidy data revealed various regional trends:

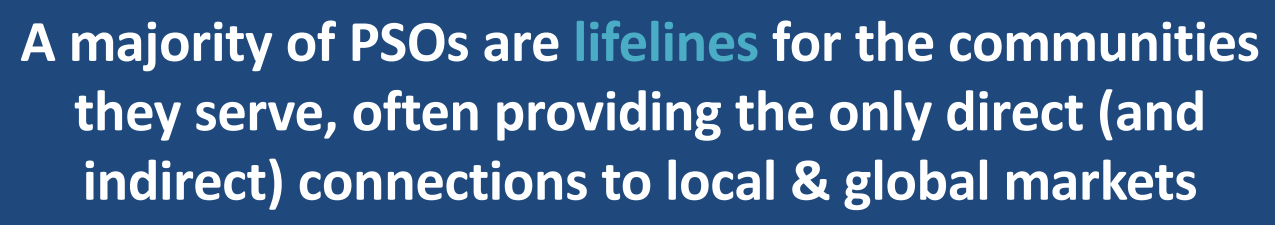

A majority of PSOs are lifelines for the communities they serve, often providing the only direct (and indirect) connections to local \& global markets

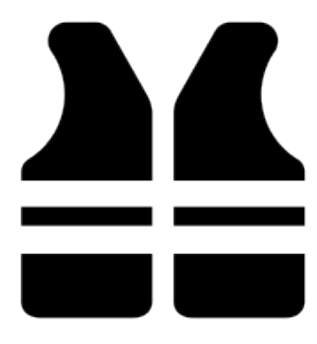

PSOs that focus on increasing indirect market access, such as those in the U.S. and Germany, provide the best connectivity "bang for buck"

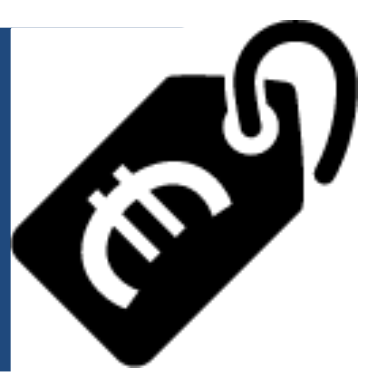

- A GCl-based analysis could be used by policymakers when choosing between different tenders for the same PSO route to determine which offer provides the best market access value for money. 


\section{For details}

The manuscript discussed in this presentation

is accepted for publication in Transportation Research Part $A$

DOI: 10.1016/j.tra.2016.08.029

A method paper on the Global Connectivity Index

was published in 2015 in Transportation Research Part E:

How air transport connects the world: A new metric for global air connectivity:

DOI: 10.1016/j.tre2015.06.001

Longer report on methodology \& connectivity trends by world-region

is available in the report series of the

MIT International Center of Air Transportation

http://hdl.handle.net/1721.1/95968 


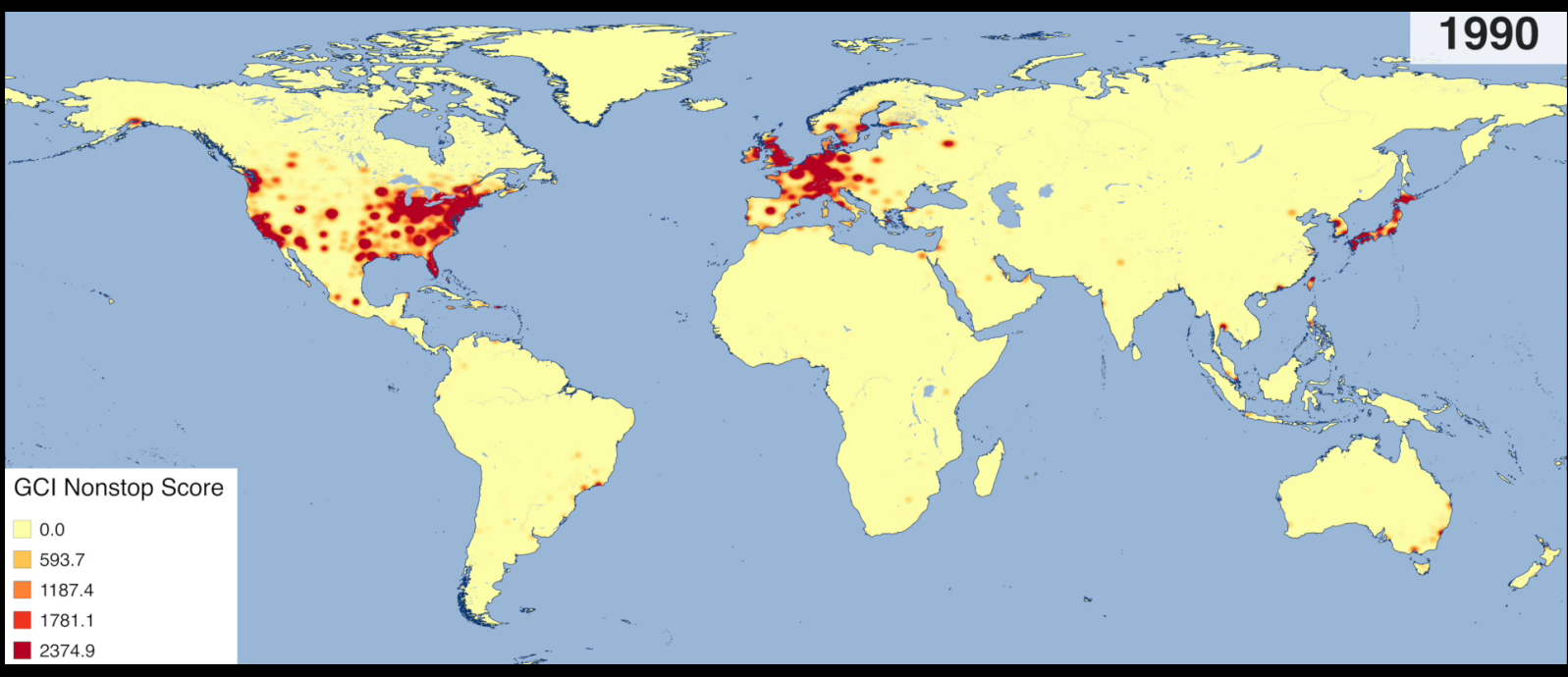

\section{Thank you!}

\section{Onestop Connectivity}

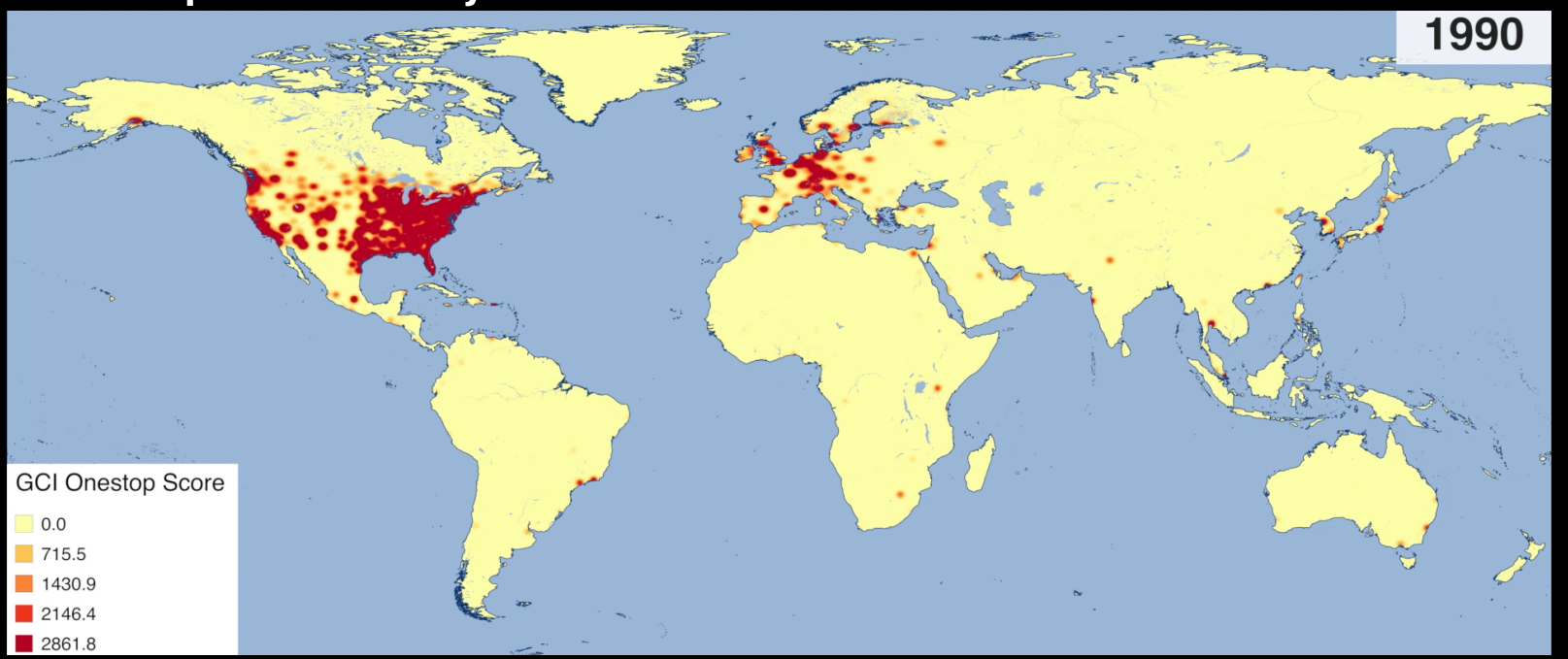

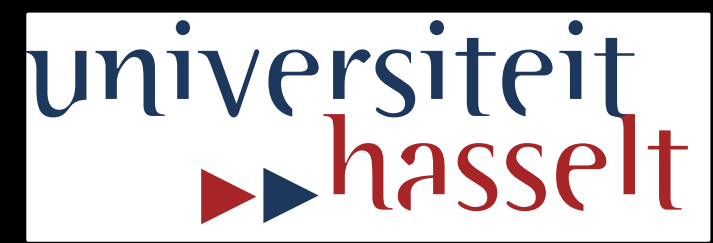

Email:

robert.malina@uhasselt.be

Florian Allroggen acknowledges funding by the German Research Foundation [DFG]. Mike Wittman acknowledges financial support by the MIT Airline Consortium. 


\section{Potential extension}

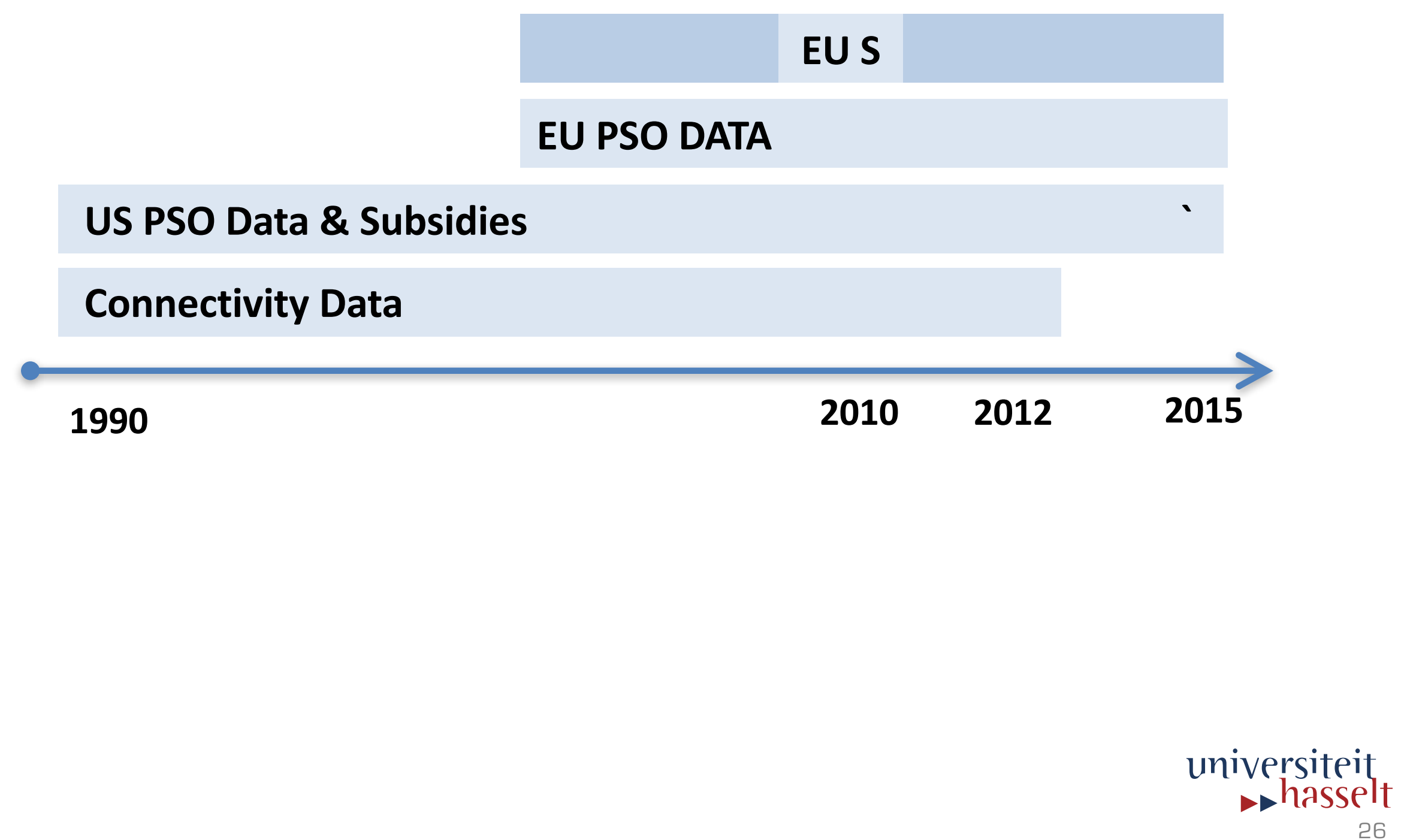


Classification of PSO Countries into Three General Archetypes

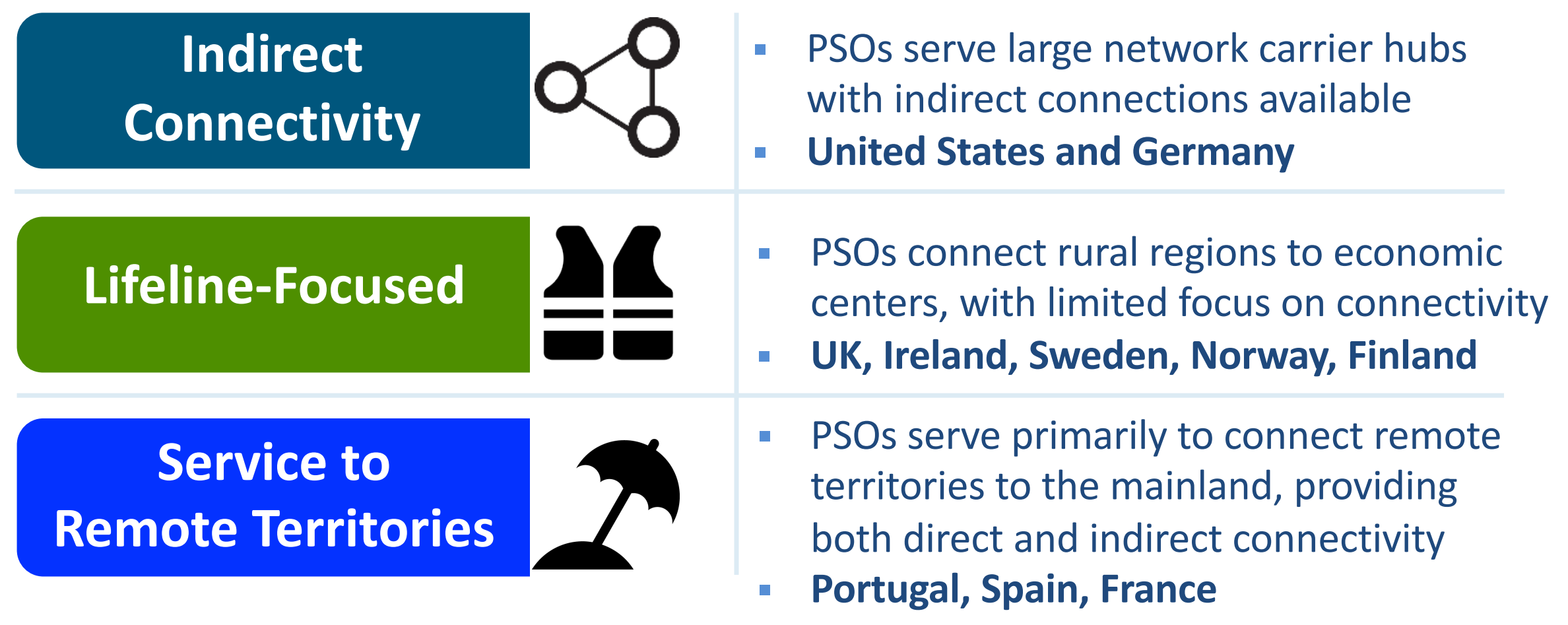




\section{Literature comparison}

\begin{tabular}{|c|c|c|c|c|c|}
\hline Study & $\begin{array}{l}\text { European } \\
\text { PSOs }\end{array}$ & US PSOs & $\begin{array}{c}\text { Subsidy } \\
\text { Data }\end{array}$ & $\begin{array}{l}\text { Multinational } \\
\text { Subsidy Data }\end{array}$ & $\begin{array}{l}\text { Connectivity } \\
\text { Focused }\end{array}$ \\
\hline Halpern and Bråthen (2011) & $\nabla$ & & & & \\
\hline Di Francesco \& Pagliari (2012) & $\nabla$ & & & & \\
\hline Williams (2012) & $\nabla$ & & & & \\
\hline Merkert and O'Fee (2013) & $\nabla$ & & & & \\
\hline Merkert and Williams (2013) & $\nabla$ & & & & \\
\hline Matisziw and Wei (2012) & & $\nabla$ & & & \\
\hline Reynolds-Feighan (1999) & $\nabla$ & $\nabla$ & & & \\
\hline Santana (2009) & $\nabla$ & $\nabla$ & & & \\
\hline Metrass-Mendes et al. (2013) & $\nabla$ & $\nabla$ & & & \\
\hline Lian et al. (2010) & $\nabla$ & & $\nabla$ & & \\
\hline Anger et al. (2012) & $\nabla$ & & $\nabla$ & & \\
\hline Williams and Pagliari (2004) & $\nabla$ & & $\nabla$ & $\nabla(7)$ & \\
\hline This work & $\nabla$ & $\nabla$ & $\nabla$ & $\nabla(11)$ & $\nabla$ \\
\hline
\end{tabular}




\section{Destination quality}

\begin{tabular}{|c|c|c|c|c|c|}
\hline Airport & City & Country & $\begin{array}{l}\text { IATA } \\
\text { Code }\end{array}$ & $w_{d_{r}, t}$ & $\begin{array}{l}\text { Percentile } \\
\text { in Dataset }\end{array}$ \\
\hline Tokyo Intl. & Tokyo & Japan & HND & 0.949 & $0.0 \%$ \\
\hline Newark Liberty Intl. & Newark & United States & EWR & 0.730 & $0.2 \%$ \\
\hline John F. Kennedy Intl. & New York & United States & JFK & 0.705 & $0.2 \%$ \\
\hline Heathrow & London & United Kingdom & LHR & 0.594 & $0.5 \%$ \\
\hline Los Angeles Intl. & Los Angeles & United States & LAX & 0.512 & $0.7 \%$ \\
\hline Düsseldorf & Düsseldorf & Germany & DUS & 0.501 & $0.8 \%$ \\
\hline Manchester & Manchester & United Kingdom & MAN & 0.445 & $1.2 \%$ \\
\hline Charles De Gaulle & Paris & France & CDG & 0.409 & $1.6 \%$ \\
\hline Schiphol & Amsterdam & Netherlands & AMS & 0.360 & $2.1 \%$ \\
\hline Chicago O'Hare Intl. & Chicago & United States & ORD & 0.360 & $2.1 \%$ \\
\hline Brussels & Brussels & Belgium & BRU & 0.359 & $2.2 \%$ \\
\hline Incheon Intl. & Seoul & South Korea & $\mathrm{ICN}$ & 0.340 & $2.5 \%$ \\
\hline Ronald Reagan Washington Natl. & Washington & United States & DCA & 0.276 & $3.2 \%$ \\
\hline Washington Dulles Intl. & Washington & United States & IAD & 0.246 & $4.1 \%$ \\
\hline Hong Kong Intl. & Hong Kong & Hong Kong & HKG & 0.221 & $4.6 \%$ \\
\hline Bahrain Intl. & Bahrain & Bahrain & BAH & 0.036 & $24.9 \%$ \\
\hline Lourdes & Tarbes & France & LDE & 0.035 & $25.1 \%$ \\
\hline Lanzarote & Las Palmas & Spain & $\mathrm{ACE}$ & 0.006 & $50.0 \%$ \\
\hline Luxor Intl. & Luxor & Egypt & LXR & 0.005 & $50.2 \%$ \\
\hline Strahan Airport & Strahan & Australia & SRN & 0.001 & $74.9 \%$ \\
\hline Teniente Vidal & Coyhaique & Chile & GXQ & 0.001 & $75.2 \%$ \\
\hline
\end{tabular}




\section{The link quality level: Route directness}

\section{Idea:}

Valuation of route directness as compared to a (hypothetical) nonstop routing between an origin airport and a destination airport.

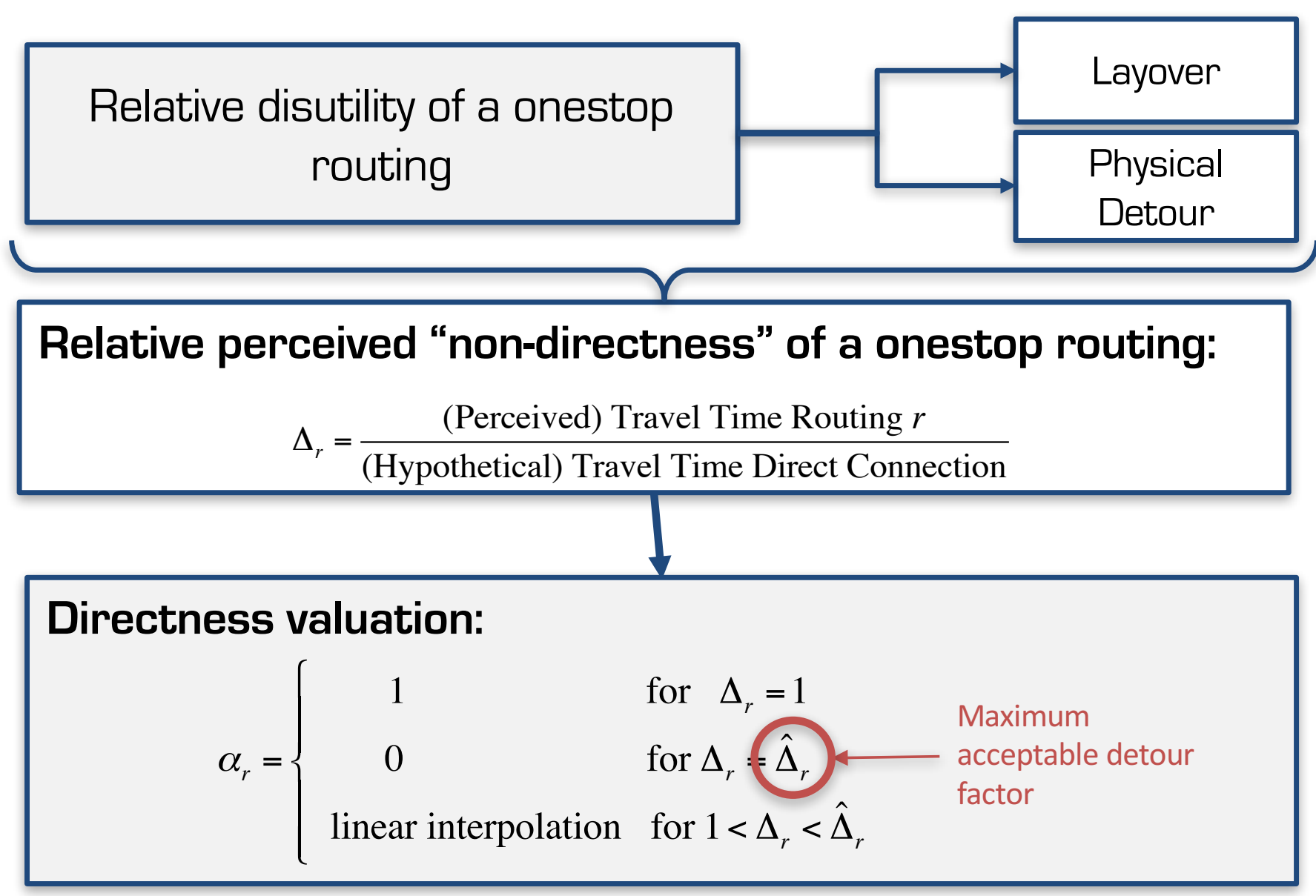



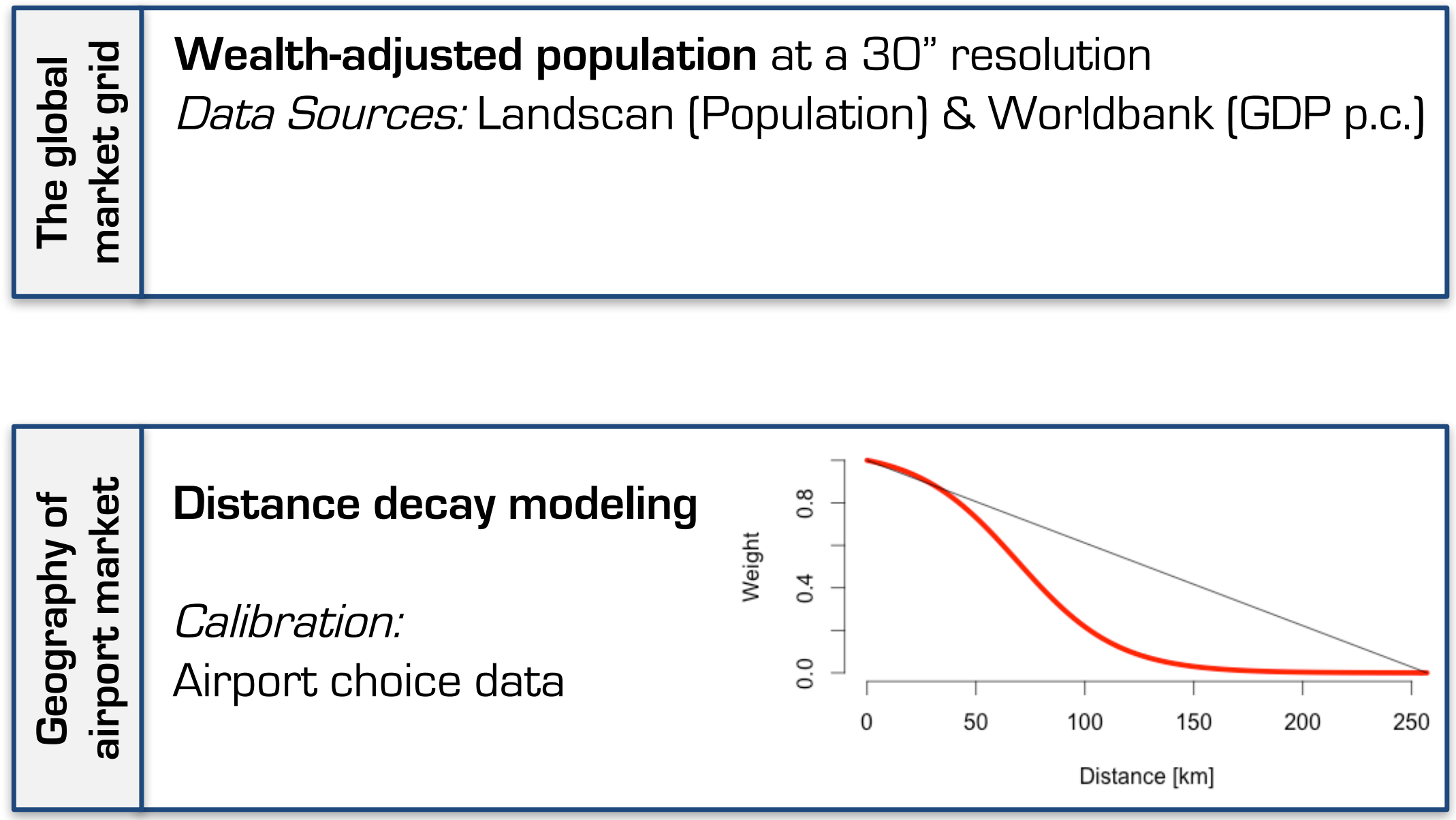
Outline

How much public money is being spent on PSO's and how does this compare to the connectivity impacts of those routes? 


\section{Outline}

To what extent do specific PSO's contribute to connectivity? 


\section{PSOs Represented About $1 \%$ of Total Connectivity}

\section{Across the 12 Countries in our Dataset}

Connectivity Impact of PSO Movements in the U.S. and Europe, 2010

\begin{tabular}{|c|c|c|c|c|c|c|c|}
\hline \multirow{2}{*}{ Country } & \multicolumn{3}{|c|}{ GCI Scores } & \multicolumn{2}{|c|}{$\%$ of Total PSO GCI } & \multirow{2}{*}{$\begin{array}{l}\text { GCI Per PSO } \\
\text { Movement }\end{array}$} & \multirow{2}{*}{$\begin{array}{c}\text { PSO \% of } \\
\text { County Total }\end{array}$} \\
\hline & Direct & Indirect & Total & Direct & Indirect & & \\
\hline Finland & 46 & 4 & 50 & $92 \%$ & $8 \%$ & 0.03 & $0.1 \%$ \\
\hline France & 5,170 & 5,416 & 10,586 & $49 \%$ & $51 \%$ & 0.25 & $4.1 \%$ \\
\hline Germany & 334 & 1,294 & 1,628 & $21 \%$ & $79 \%$ & 0.63 & $0.4 \%$ \\
\hline Greece & 340 & 363 & 703 & $48 \%$ & $52 \%$ & 0.05 & $1.5 \%$ \\
\hline Ireland & 436 & 70 & 506 & $86 \%$ & $14 \%$ & 0.05 & $1.0 \%$ \\
\hline Italy & 2,156 & 1,110 & 3,266 & $66 \%$ & $34 \%$ & 0.10 & $1.2 \%$ \\
\hline Norway & 778 & 96 & 874 & $89 \%$ & $11 \%$ & 0.02 & $1.5 \%$ \\
\hline Portugal & 218 & 748 & 966 & $23 \%$ & $77 \%$ & 0.05 & $2.5 \%$ \\
\hline Spain & 1,218 & 1,082 & 2,300 & $53 \%$ & $47 \%$ & 0.03 & $1.0 \%$ \\
\hline Sweden & 118 & 0 & 118 & $100 \%$ & $0 \%$ & 0.02 & $0.2 \%$ \\
\hline U.K. & 108 & 143 & 251 & $43 \%$ & $57 \%$ & 0.01 & $0.1 \%$ \\
\hline U.S. & 11,336 & 41,448 & 52,785 & $21 \%$ & $79 \%$ & 0.32 & $1.0 \%$ \\
\hline Total & 22,212 & 51,770 & 73,983 & $30 \%$ & $70 \%$ & 0.16 & $1.0 \%$ \\
\hline
\end{tabular}




\section{Future applications of connectivity metric}
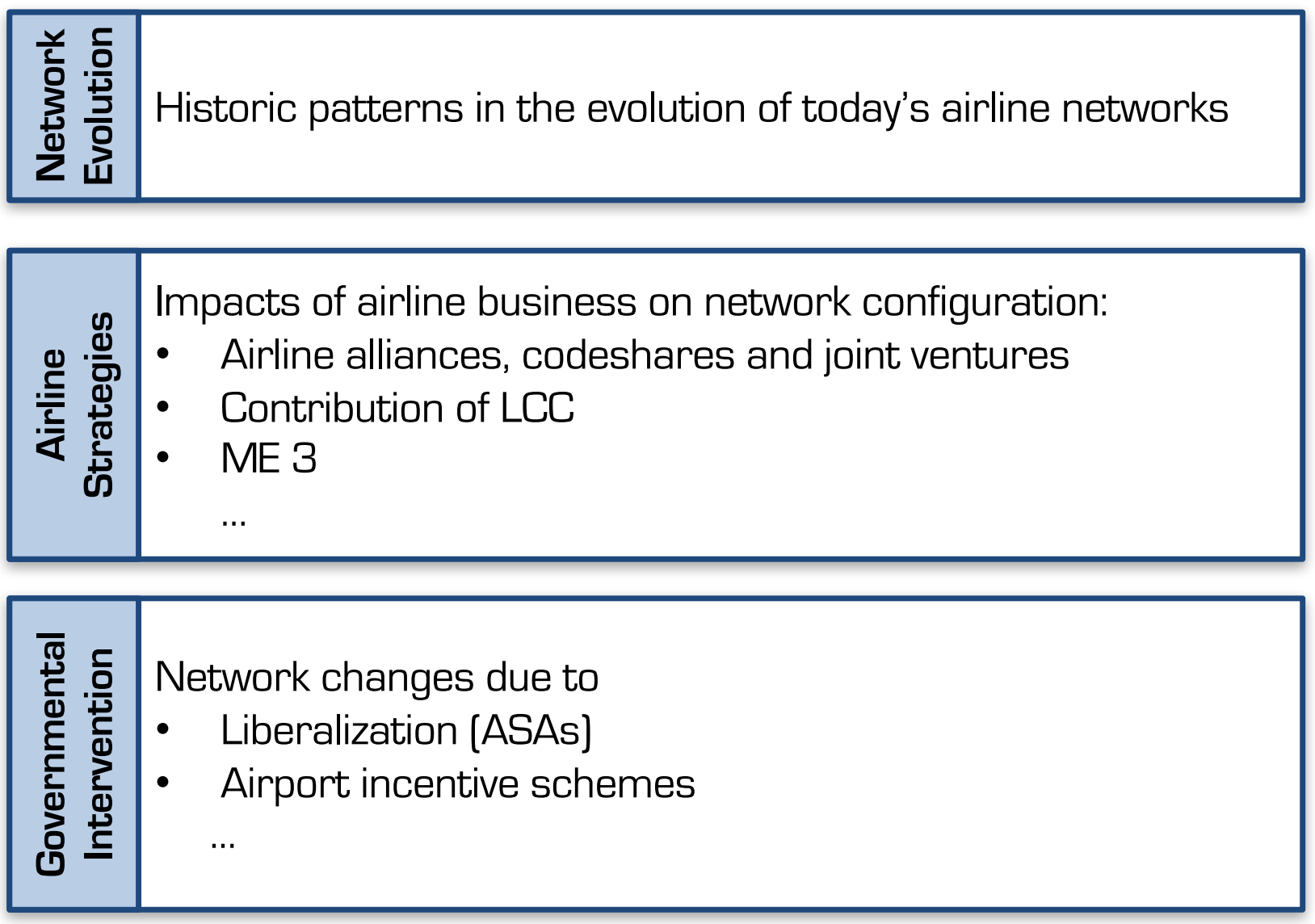

\begin{tabular}{|c|c|}
\hline 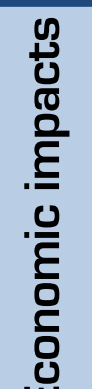 & $\begin{array}{l}\text { Connectivity impacts on: } \\
\text { - } \quad \text { Economic growth } \\
\text { - } \quad \text { Employment } \\
\text { - } \quad \text { Trade } \\
\text { - } \quad \text { Foreign Direct Investment }\end{array}$ \\
\hline
\end{tabular}

